\title{
Local-structure analysis around dopant atoms using multiple energy $\mathrm{x}$-ray holography
}

\section{$\operatorname{AUTHOR}(\mathrm{S})$ :}

Hayashi, K; Matsui, M; Awakura, Y; Kaneyoshi, T; Tanida, H; Ishii, M

\section{CITATION:}

Hayashi, K ...[et al]. Local-structure analysis around dopant atoms using multiple energy x-ray holography. PHYSICAL REVIEW B 2001, 63(4): 041201.

\section{ISSUE DATE:}

2001-01-15

URL:

http://hdl.handle.net/2433/39843

RIGHT:

Copyright 2001 American Physical Society 


\title{
Local-structure analysis around dopant atoms using multiple energy x-ray holography
}

\author{
Kouichi Hayashi, ${ }^{1}$ Masaki Matsui, ${ }^{1}$ Yasuhiro Awakura, ${ }^{1}$ Takahiro Kaneyoshi, ${ }^{2}$ Hajime Tanida, ${ }^{3}$ and Masashi Ishii ${ }^{3}$ \\ ${ }^{1}$ Department of Materials Science and Engineering, Kyoto University, Sakyo-ku, Kyoto, Japan \\ ${ }^{2}$ Hyogo Prefectural Institute of Industrial Research, Suma-ku, Kobe, Japan \\ ${ }^{3}$ Japan Synchrotron Research Institute, SPring-8, Mikazuki-cho, Sayo-gun, Hyogo, Japan
}

(Received 8 September 2000; published 9 January 2001)

\begin{abstract}
We used multiple energy $\mathrm{x}$-ray holography (MEXH) to image the local atomic environment of $\mathrm{Zn}$ atoms doped in a GaAs wafer using synchrotron radiation and a multielement solid-state detector. The obtained atomic images revealed that the $\mathrm{Zn}$ atoms occupied substitutional site. By the comparison of the reconstructed images $1.41 \AA$ above and below the emitter atom, the suppression of twin images due to MEXH was confirmed experimentally for certain atoms.
\end{abstract}

DOI: 10.1103/PhysRevB.63.041201

PACS number(s): 61.10.-i, 07.85.Qe, 42.40.-i

\section{INTRODUCTION}

$\mathrm{X}$-ray fluorescence holography (XFH) is a technique which can record the amplitude and phase of wavefronts scattered by atoms, relative to direct unscattered waves, and directly provide a three-dimensional atomic image of the environment around the atoms emitting the fluorescence. ${ }^{1}$ Two types of this method have been developed: "normal XFH" and "inverse XFH.' In normal XFH, fluorescence from atoms inside the sample approaching the detector on a direct path constitutes the holographic reference beam, and fluorescence scattered by neighboring atoms acts as the object beam. ${ }^{2-7}$ A holographic pattern can be recorded moving the detector around the sample. The inverse XFH is based on the idea of the optical reciprocity of the normal XFH and the application of known principles for $\mathrm{x}$-ray standing waves. The atoms emitting fluorescence serve as the detector of the interference field originating from the incident and scattered $\mathrm{x}$ rays, which constitutes the reference and object beams, respectively. The holographic pattern can be obtained by detecting the fluorescence while varying the sample orientation relative to the incident beam. Since the incident $x$ rays can be of any energy above the absorption edge of the emitter, inverse XFH allows holograms to be recorded at an arbitrary energy, which can suppress the twin-image effect. ${ }^{8}$ In general, this method is called "multiple energy x-ray holography' (MEXH).

Until now, most of the papers about XFH have reported demonstrations for structural determination of single crystals, whose atomic configuration was already known by the x-ray-diffraction method. Gog et al. tried to determine the structure of $\mathrm{GaP}$ small clusters in the $\mathrm{Si}$ matrix using MEXH. ${ }^{9}$ In 1998, we measured the hologram of $\mathrm{Zn}$ doped in a GaAs wafer by the single energy inverse XFH and successfully obtained an atomic image, suggesting that the $\mathrm{Zn}$ atoms occupied the substitutional site. ${ }^{10}$ However, the obtained atomic image was blurry and distorted, because the $S / N$ of the hologram data was low due to an insufficient total count of the fluorescence in each pixel of the hologram. In order to check the reproducibility of the above results and to evaluate the feasibility of MEXH for structural analysis around the impurity element, we again carried out the holography experiment of $\mathrm{Zn}$ in GaAs at two different energies using syn- chrotron radiation. In this experiment, a multielement solidstate detector (SSD) was used for recording the hologram. The multielement SSD is suitable for the inverse XFH of the impurity element, because it has a large detecting area and enables high countrate measurement.

\section{EXPERIMENTAL DETAILS}

The hologram measurement was carried out using synchrotron beam line BL10XU at SPring-8 in October 1999. SPring-8 (Super Photon ring- $8 \mathrm{GeV}$ ) is one of the ultrahighbrilliance synchrotron facilities in the world. The electron storage ring current was between 100 and $80 \mathrm{~mA}$ during measurements. The synchrotron radiation from an undulator was monochromatized by a Si (111) double-crystal monochromator. The GaAs:Zn wafer (001) was purchased from Furuuchi Chemical Co. (Tokyo, Japan). The Zn concentration in the wafer was determined to be 1.0 $\times 10^{19}$ atoms $\mathrm{cm}^{-3}(0.02 \mathrm{wt} \%)$ by a Hall measurement. The diameter and thickness of the sample were 50.0 and 0.25 $\mathrm{mm}$, respectively. The incident $\mathrm{x}$-ray energy was 9.7 and $10.0 \mathrm{keV}$, which was between the $\mathrm{Zn}$ and $\mathrm{Ga} K$ absorption edges, so as to avoid excitation of the Ga and As x-ray fluorescence.

Figure 1 shows a schematic illustration of the experimental setup. The sample was mounted on a two-axis $(\theta-\phi)$ rotatable stage, where $\theta$ was the polar angle between the incident beam and the surface normal, and $\phi$ was the azimuthal angle between the [110] and the projection of the incident beam. The [110] direction of the sample was determined from the cleavage planes. Multielement SSD was placed parallel to the incident $\mathrm{x}$-ray electric field, as shown in Fig. 1. The intensity of $\mathrm{Zn} K \alpha$ x-ray fluorescence was measured as a function of the azimuthal angle, $\phi$, and polar angle, $\theta$, within the ranges of $0^{\circ} \leqslant \phi \leqslant 360^{\circ}$ and $26^{\circ} \leqslant \theta \leqslant 60^{\circ}$. The dwelling time for one pixel was $1 \mathrm{~s}$ with $2^{\circ}$ steps in $\theta$ and $1^{\circ}$ steps in $\phi$. The total integrated intensity of the $\mathrm{Zn} K \alpha$ x-ray fluorescence at each pixel was about 200000 counts.

\section{RESULTS AND DISCUSSION}

Since the raw hologram data were noisy, they were smoothed by applying low and high pass filters. The holo- 


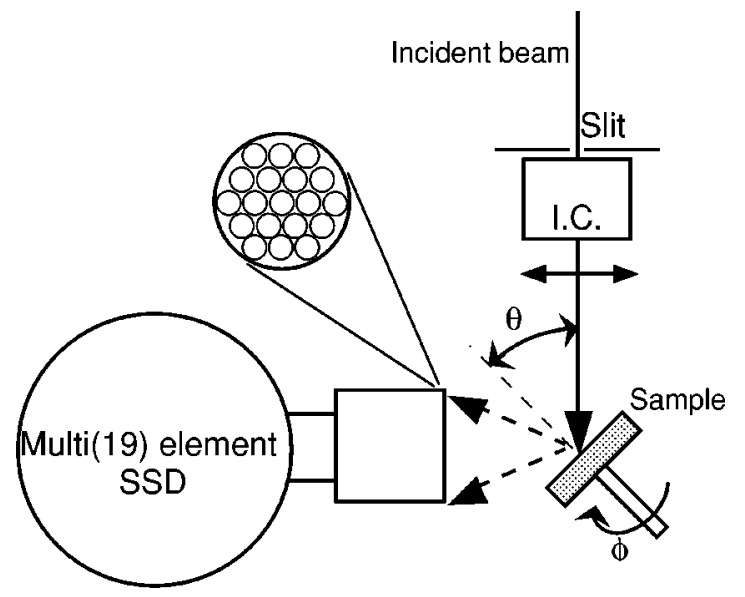

FIG. 1. Experimental setup for MEXH measurement. The incident beam size was $0.25 \times 0.25 \mathrm{~mm}^{2}$ and its intensity was monitored by an ionization chamber. X-ray fluorescence was detected by a 19 element solid-state detector. Angular acceptance of the detecting area was approximately $43^{\circ}$. The incident beam was linearly polarized and the electric field was parallel to the horizontal plane. The experiment was performed using the BL10XU beamline of SPring-8.

graphic patterns for the incident energies of 9.7 and $10.0 \mathrm{keV}$ were different, while they showed periodical undulation reflecting the symmetry of the GaAs (001) plane. We reconstructed the atomic image from these holograms using the Barton algorithm, ${ }^{11}$ and successfully obtained the images of the first- and second-neighbor atoms around the emitter. But, the reconstructed atomic images were distorted and no more atoms could be observed.

In order to obtain clearer atomic images, we symmetrized the holograms by the use of the symmetry of the host GaAs single crystal. $^{12}$ Figures 2(a) and 2(b) show twofoldaveraged holographic patterns for 9.7 and $10.0 \mathrm{keV}$, respectively. From these holograms, we again reconstructed atomic images of planes parallel to the $\{001\}$ lattice plane cutting through the $\mathrm{Zn}$ atom, and $1.41 \AA$ ( $\cong / 4)$ above and below the emitter atom, which are termed here planes $A, B$, and $C$. Figures 3(a), 3(b), and 3(c) show the reconstructed images of planes $A, B$, and $C$. From Fig. 3(a), 110, $\overline{1} 10,1 \overline{1} 0$, and $\overline{1} 10$ atoms are clearly seen and the distances between the intensity maxima of these atoms and the emitter are equally 4.02 $\AA$. The crystal structure of $\mathrm{GaAs}$ is a $\mathrm{ZnS}$-like structure with $a=5.65 \AA$, that is, it consists of two face-centered-cubic cells. The Ga and As layers stack alternately along the $c$ axis; these two layers are separated by $1.41 \AA$. The atomic configuration of the Ga layer is the same as that of the As layer, and the nearest Ga-Ga or As-As distances are 4.00 А. Thus, the $\mathrm{Zn}$ atoms are found to substitute for a Ga or As site. This result well agreed with extended x-ray_absorption fine structure (EXAFS) one. ${ }^{13} 200, \overline{2} 00,020,0 \overline{2} 0$ atoms are also visible at distances of $7.02 \AA$, which is $25 \%$ larger than the distance $(5.65 \AA$ ) expected from the GaAs crystal structure.

In Fig. 3(b), strong $\frac{1}{2} \frac{1}{2} \frac{1}{4}, \overline{\frac{1}{2}} \frac{1}{2} \frac{1}{4}, \frac{3}{2} \frac{3}{2} \frac{1}{4}, \frac{\overline{3}}{2} \frac{3}{2} \frac{1}{4}$ atoms and weak $\frac{1}{2} \frac{1}{2} \frac{1}{4}, \frac{1}{2} \frac{1}{2} \frac{1}{4}, \frac{\overline{3}}{2} \frac{3}{2} \frac{1}{4}, \frac{3}{2} \frac{\overline{3}}{2} \frac{1}{4}$ atoms are seen, revealing that $\mathrm{Zn}$ atoms are substituted selectively for one site of $\mathrm{Ga}$ or As. The possibility of As-site substitution may be negligible
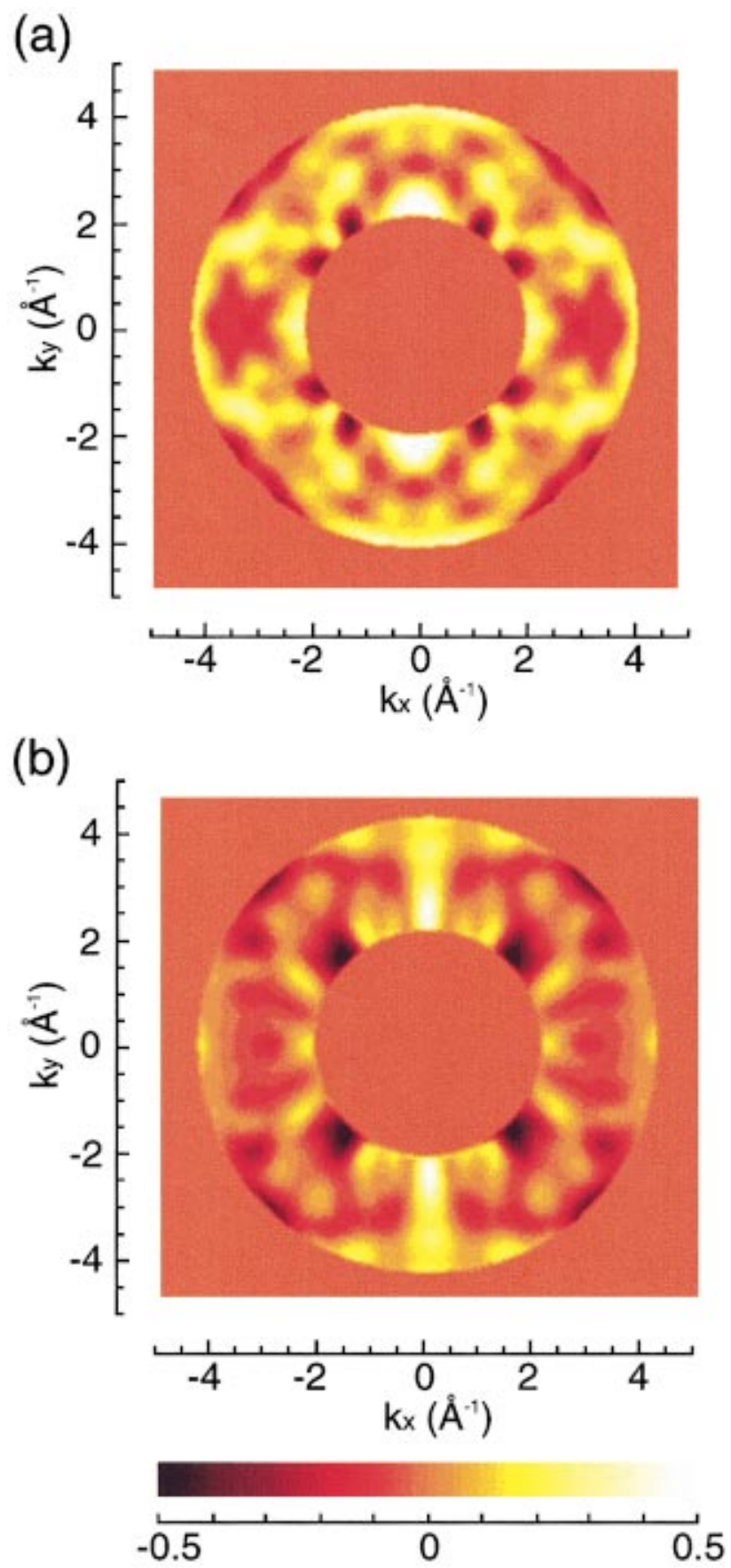

FIG. 2. (Color) Holograms of $\mathrm{Zn}$ in GaAs recorded at the incident energies of (a) $9.7 \mathrm{keV}$ and (b) $10.0 \mathrm{keV}$. Hologram data $\chi^{\prime}(\theta, \phi)$ were twofold averaged using the relation $\chi^{\prime}(\theta, \phi)=\left[\chi(\theta, \phi)+\chi\left(\theta, \phi+180^{\circ}\right)+\chi(\theta,-\phi)+\chi\left(\theta,-\phi+180^{\circ}\right)\right] / 4$, where $\chi(\theta, \phi)$ was hologram data after applying the low and high pass filters. $\chi^{\prime}(\theta, \phi)$ was projected onto the $k x$ - $k y$ plane, where $k x=|k| \cos \phi \sin \theta, k y=|k| \sin \phi \sin \theta$ and $|k|$ is the wave vector magnitude of incident $\mathrm{x}$ rays.

because of the charge neutrality. Figure 4 shows the possible model of the atomic arrangement around $\mathrm{Zn}$ atoms. Since the intensity of the $\mathrm{x}$ rays scattered from the atoms lying on plane $B$ is stronger than those on plane $C$, it is considered that the $\frac{1}{2} \frac{1}{2} \frac{1}{4}, \overline{\frac{1}{2}} \frac{1}{2} \frac{1}{4}, \frac{3}{2} \frac{3}{2} \frac{1}{4}, \frac{3}{2} \frac{3}{2} \frac{1}{4}$ atoms are the real image 
(a)

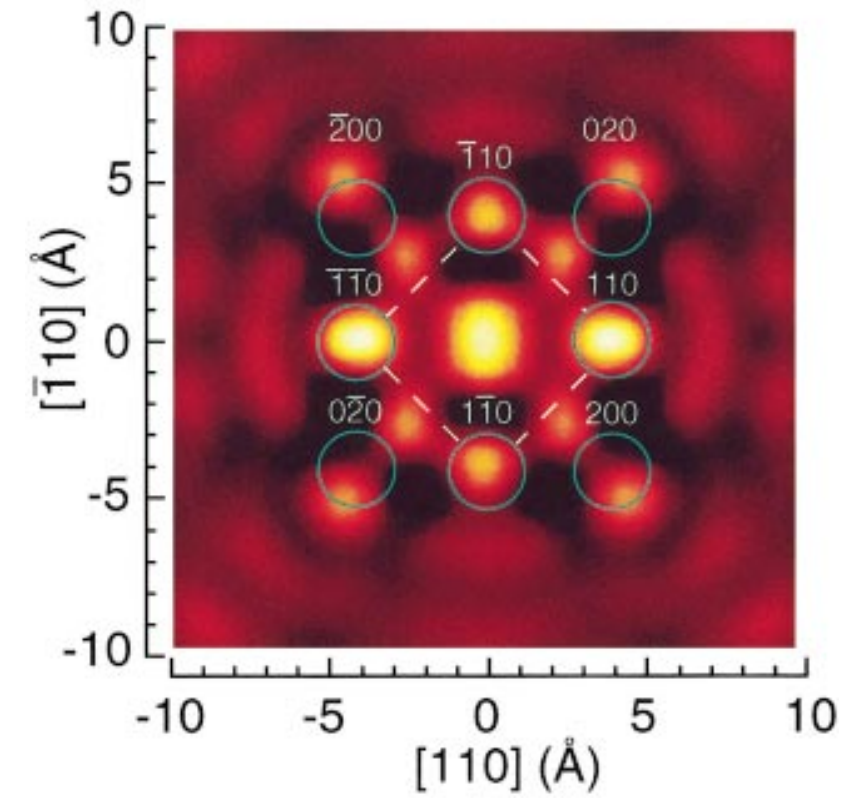

(b)

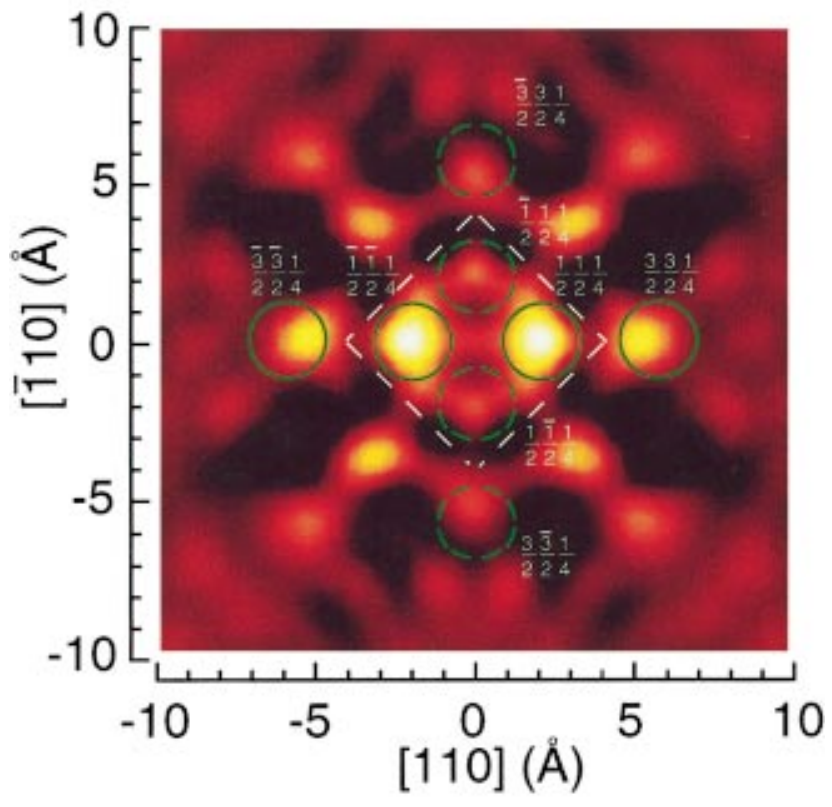

(c)

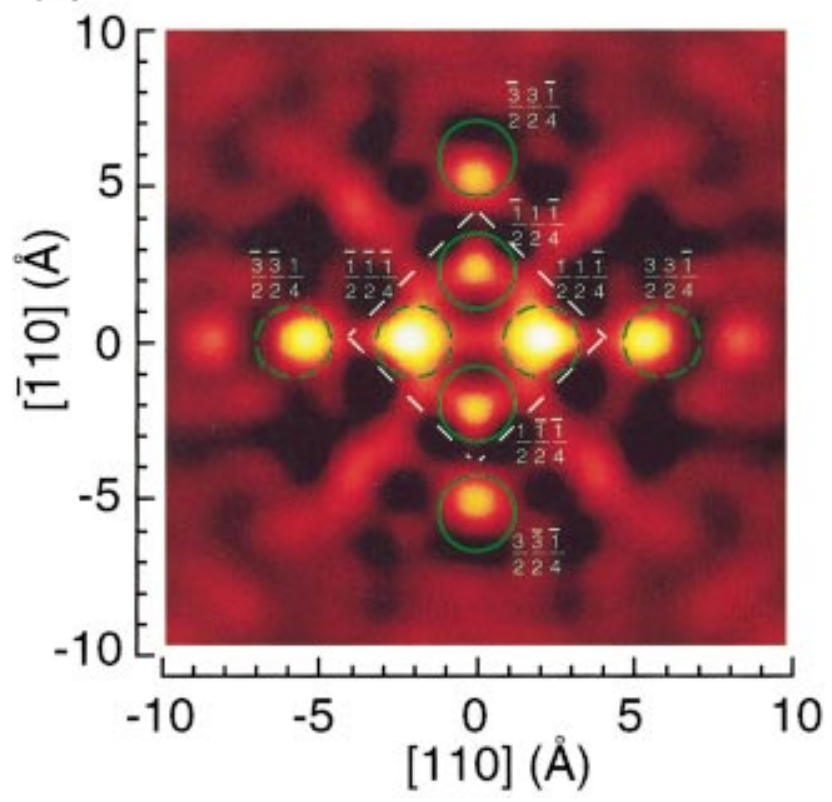

FIG. 3. (Color) Holographic reconstruction of an environment around the $\mathrm{Zn}$. The planes parallel to the $\{001\}$ lattice plane cutting through the fluorescence emitter atom, $1.41 \AA$ above and below the emitter atom are displayed in (a), (b), and (c), respectively. The dashed lines show the outline of the GaAs crystal cell. Solid and dotted circles show real and twin images, respectively.

and that $\frac{1}{2} \frac{1}{2} \frac{1}{4}, \frac{1}{2} \frac{1}{2} \frac{1}{4}, \frac{3}{2} \frac{3}{2} \frac{1}{4}, \frac{3}{2} \frac{3}{2} \frac{1}{4}$ atomlike images are twin images of the $\frac{1}{2} \frac{1}{2} \frac{1}{4}, \frac{1}{2} \frac{1}{2} \frac{1}{4}, \frac{3}{2} \frac{3}{2} \frac{1}{4}, \frac{3}{2} \frac{\overline{3}}{2} \frac{1}{4}$ atoms existing on plane $C$, respectively. Holographic twin images appear necessarily in the image reconstructed from the single energy hologram and are suppressed by the reconstruction from the MEXH data. Twin image suppression becomes effective with an increase in the number of holograms recorded at different energies. However, since we measured only two holograms in our experiment, this effect is considered to be weak.
The $\frac{1}{2} \frac{1}{2} \frac{1}{4}, \frac{1}{2} \frac{1}{2} \frac{1}{4}, \frac{3}{2} \frac{3}{2} \frac{1}{4}, \frac{3}{2} \frac{3}{2} \frac{1}{4}$ atomic images appearing in Fig. 3(c) are twin images of $\frac{1}{2} \frac{1}{2} \frac{1}{4}, \frac{1}{2} \frac{1}{2} \frac{1}{4}, \frac{3}{2} \frac{3}{2} \frac{1}{4}, \frac{3}{2} \frac{3}{2} \frac{1}{4}$, atoms in Fig. 3(b), respectively. Since the intensity of each twin image nearly equaled that of the paired real image, the twin image suppression was not confirmed for these atoms. In contrast, the real images of $\frac{1}{2} \frac{1}{2} \frac{1}{4}, \frac{1}{2} \frac{1}{2} \frac{1}{4}, \frac{3}{2} \frac{3}{2} \frac{1}{4}, \frac{3}{2} \frac{3}{2} \frac{1}{4}$ atoms in Fig. 3(c) are obviously intensive compared to their twin images displayed in Fig. 3(b). This result revealed that two energy MEXH data contribute to suppressing the twin 
image for $\frac{\overline{1}}{2} \frac{1}{2} \frac{1}{4}, \frac{1}{2} \frac{1}{2} \frac{1}{4}, \frac{\overline{3}}{2} \frac{3}{2} \overline{\frac{1}{4}}, \frac{3}{2} \frac{\overline{3}}{2} \frac{1}{4}$ atoms. In addition to these, atomlike images are observed at $(x, y)=( \pm 2.6 \AA$, $\pm 2.6 \AA)$ or $( \pm 5.2 \AA, \pm 5.2 \AA)$ on plane $B$ and the intensities of their twin image on plane $C$ are also suppressed, but we could not assign them in this study.

The distances from the center to $\frac{1}{2} \frac{1}{2} \frac{1}{4}, \overline{\frac{1}{2}} \frac{1}{2} \frac{1}{4}, \frac{1}{2} \frac{1}{2} \frac{1}{4}$, $\frac{1}{2} \frac{1}{2} \frac{1}{4}$ atoms are equally $1.94 \AA$ which well agreed with the distance $(2.00 \AA)$ predicted from the knowledge on the EXAFS and crystal data. While the distances from the center to $\frac{3}{2} \frac{3}{2} \frac{1}{4}, \frac{3}{2} \frac{3}{2} \frac{1}{4}, \overline{3} \frac{3}{2} \frac{3}{4}, \frac{3}{2} \frac{3}{2} \frac{1}{4}$ atoms are equally $5.13 \AA$, which is $14 \%$ shorter than the expected distance $(6.00 \AA)$. Taking into account the image of plane $A$, the atomic images of first- and second-neighbor atoms within the radius of $4 \AA$ appear at accurate positions, while the positions of the other atomic images are largely displaced. It is considered that these large displacements originate from the weakness of the holographic signal from the atoms existing at the distance of over $4 \AA$ from the emitter. This problem will be resolved by the increase of the total count in each pixel.

Beside imaging planes $A, B$, and $C$, the reconstruction was performed for the planes parallel to the $\{001\}$ lattice plane $2.83 \AA(\cong z / 2)$ above and below the emitter atom. The atoms existing on these planes were not visible, however, which was attributed to loss of image information along the $c$ axis as a direct consequence of the limited $k z$ range of the measured hologram as well as the polarization effect of the incident beam. When the polarization vector was perpendicular to the polar rotation axis $\theta$, the horizontal atomic structure near the basal plane was emphasized and the vertical atomic structure near the [001] axis was diminished. ${ }^{14}$ Images of these planes should become further resolved with full $2 \pi$ scans used for the incident beams of the vertical and horizontal polarizations.

\section{CONCLUSIONS}

We measured the hologram at two different energies using multielement SSD. Symmetrized holograms provide a clear atomic image around $\mathrm{Zn}$, which revealed that most of the $\mathrm{Zn}$ atoms occupied the substitutional site in our GaAs wafer. Furthermore, twin image suppression due to the

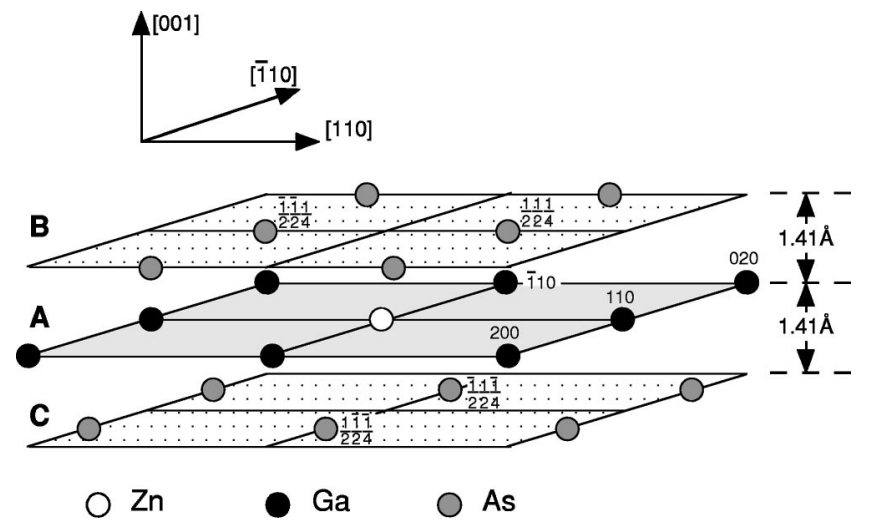

FIG. 4. Model of local environment around $\mathrm{Zn}$ atom. The atomic arrangement is obtained from the reconstructed images in Fig. 3.

MEXH was recognized for certain atoms. The centrosymmetric atomic structure images reconstructed from singleenergy holograms often diminish due to the real-twin image overlap problem. ${ }^{15}$ Therefore, the MEXH was predicted to be useful for the reconstruction of all near-neighboring atoms surrounding the emitter, and it was confirmed experimentally. $8,9,12,16,17$ Besides, the twin image suppression for non-centrosymmetric atomic structures is also important as demonstrated by Barton ${ }^{11}$ and Adams et al. ${ }^{18}$ However, it had not been confirmed experimentally as far as we know. Thus, we believe that this is experimental evidence. The present results demonstrate that the x-ray holography technique is applicable to practical structural analysis of trace impurities for a reasonable measurement time using a thirdgeneration synchrotron radiation facility.

\section{ACKNOWLEDGMENTS}

This work was performed under the approval of the SPring-8 Program Advisory Committee (1999B0121-ND$n p$ ). Part of this work was financially supported by a Grantin-Aid for Scientific Research from the Ministry of Education, Science, Sports and Culture, Japan. We thank Professor S. Hayakawa for his technical help.
${ }^{1}$ A. Szöke, Short Wavelength Coherent Radiation: Generation and Applications, edited by D. T. Attwood and J. Boker, AIP Conf. Proc. No. 147 (AIP, New York, 1986), p. 361.

${ }^{2}$ M. Tegze and G. Faigel, Europhys. Lett. 16, 41 (1991).

${ }^{3}$ M. Tegze and G. Faigel, Nature (London) 380, 49 (1996).

${ }^{4}$ P. M. Len et al., Phys. Rev. B 50, 11275 (1994).

${ }^{5}$ J. Kawai et al., Anal. Sci. 14, 903 (1998).

${ }^{6}$ K. Hayashi et al., Jpn. J. Appl. Phys. Part 1 39, 1414 (2000).

${ }^{7}$ T. Hiort et al., Phys. Rev. B 61, R830 (2000).

${ }^{8}$ T. Gog et al., Phys. Rev. Lett. 76, 3132 (1996).

${ }^{9}$ T. Gog et al., J. Electron Spectrosc. Relat. Phenom. 92, 123
(1998).

${ }^{10}$ K. Hayashi et al., Anal. Sci. 14, 987 (1998).

${ }^{11}$ J. J. Barton, Phys. Rev. Lett. 67, 3106 (1991).

${ }^{12}$ M. Tegze et al., Phys. Rev. Lett. 82, 4847 (1999).

${ }^{13}$ T. Kitano, H. Watanabe, and J. Matsui, Appl. Phys. Lett. 54, 2201 (1989).

${ }^{14}$ P. M. Len et al., Phys. Rev. B 56, 1529 (1997).

${ }^{15}$ P. M. Len et al., Phys. Rev. B 55, R3323 (1997).

${ }^{16}$ D. V. Novikov et al., J. Synchrotron Radiat. 5, 315 (1998).

${ }^{17}$ B. Adamas et al., Phys. Status Solidi B 215, 757 (1999).

${ }^{18}$ B. Adams et al., Phys. Rev. B 57, 7526 (1998). 